\title{
A Background Assessable and Correctable Bimolecular Fluorescence Complementation System for Nanoscopic Single-Molecule Imaging of Intracellular Protein-Protein Interactions
}

Shiqi Mao ${ }^{1,2, \# \text {, Yachen Ying }}{ }^{1,2, \#}$, Zhao Ma ${ }^{1, \#}$, Yantao Yang ${ }^{1,2}$, and Antony K. Chen ${ }^{1, *}$

${ }^{1}$ Department of Biomedical Engineering, College of Future Technology, Peking University, Beijing 100871, China

${ }^{2}$ Department of Biomedical Engineering, College of Engineering, Peking University, Beijing 100871, China

"S.M., Y.Y., and Z.M. contributed equally.

*ADDRESS FOR CORRESPONDENCE:

ANTONY K. CHEN

Department of Biomedical Engineering

College of Future Technology, Peking University

No. 5 Yiheyuan Road.

Haidian District, Beijing 100871, China

Tel/Fax: +861062768343

EMAIL: $\underline{\text { chenak@pku.edu.cn }}$ 
pCMVnl-Gag-VN-mCherry

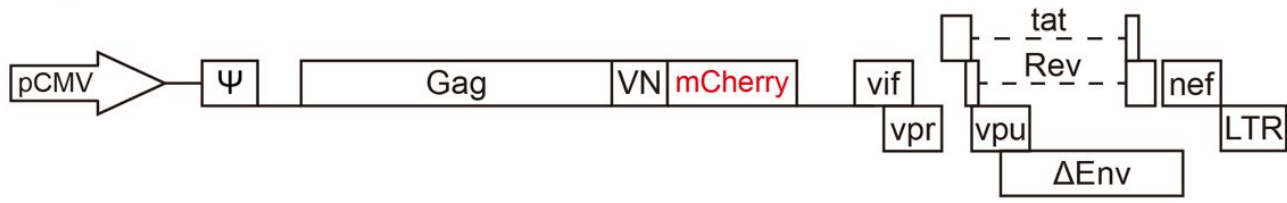

pCMVnl-Gag-VC

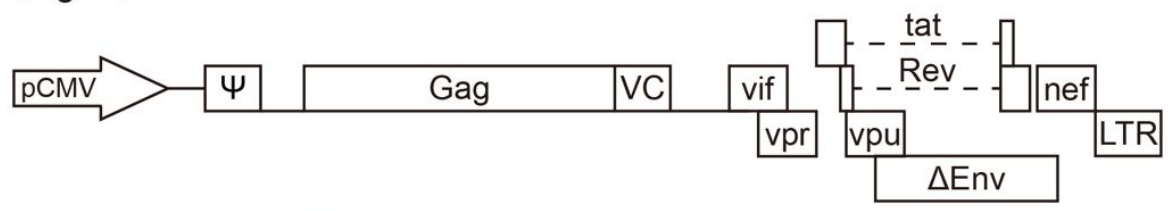

pCMVnl- $\triangle$ NC-Gag-VN-mCherry
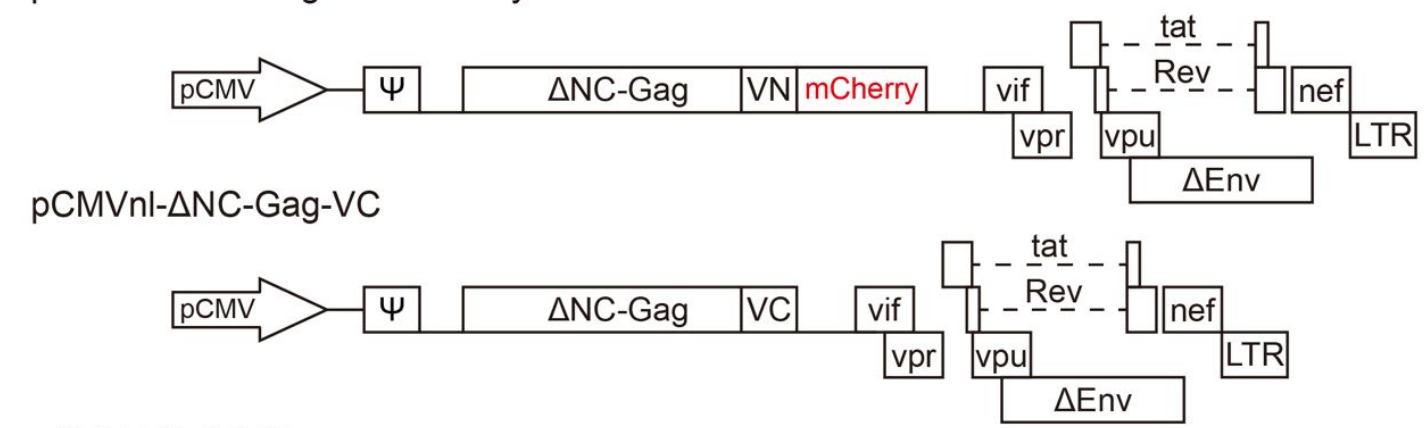

pCMV-VC-AGO2

\begin{tabular}{|l|l|}
\hline $\mathrm{PCMV}>\mathrm{VC}$ & $\mathrm{AGO} 2$ \\
\hline
\end{tabular}

Figure S1. Schematics of BAC-BiFC constructs used to image Gag-Gag interaction or Gag-AGO2 interaction. 

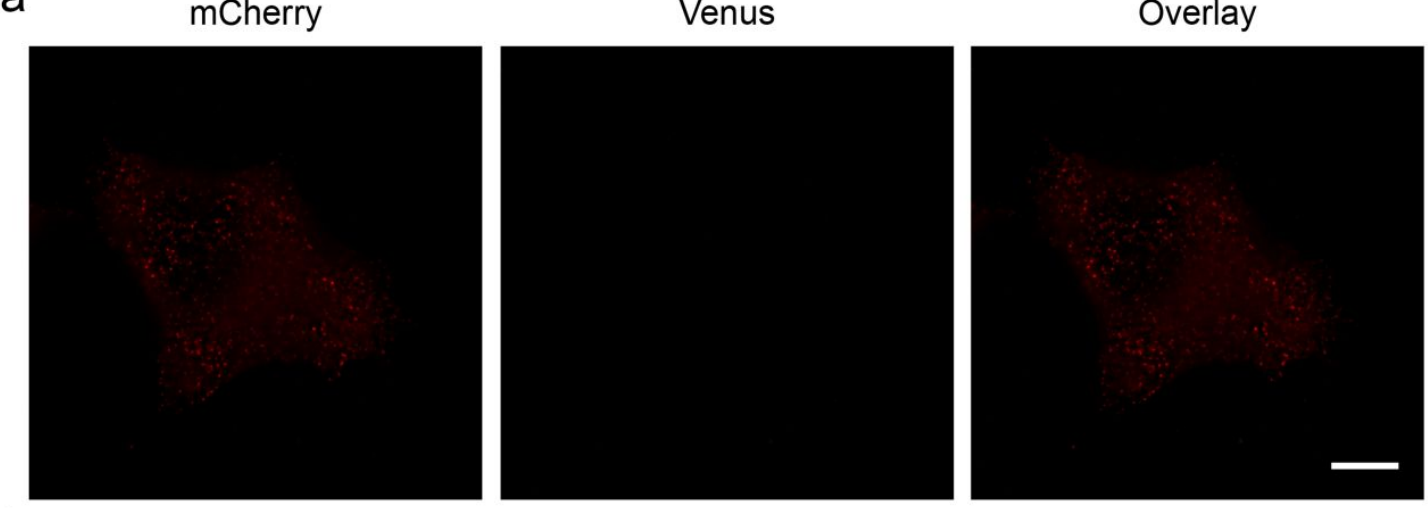

b

mCherry

Venus

Overlay
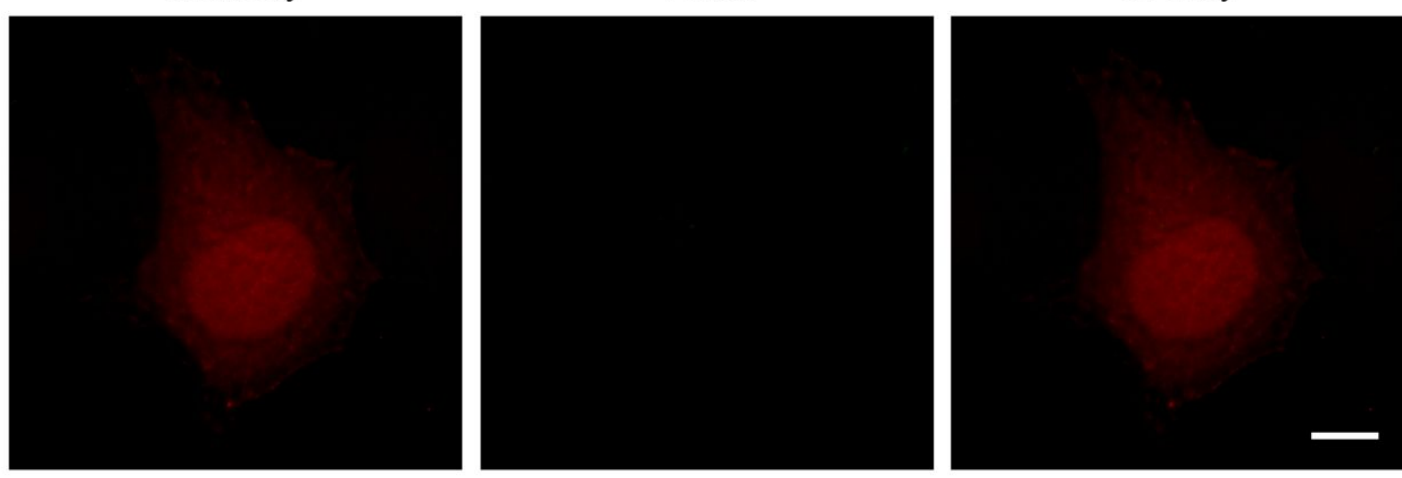

Figure S2. Widefield fluorescence imaging of HeLa cells transfected with (a) 200 ng of pCMVnl-Gag-VN-mCherry or (b) $200 \mathrm{ng}$ of pCMVnl-Gag-VC plus $20 \mathrm{ng}$ of a CMV-driven plasmid encoding mCherry fluorescence protein as a transfection indicator. Representative maximum intensity projection images of mCherry and Venus signals are shown. Scale bar, $10 \mu \mathrm{m}$.
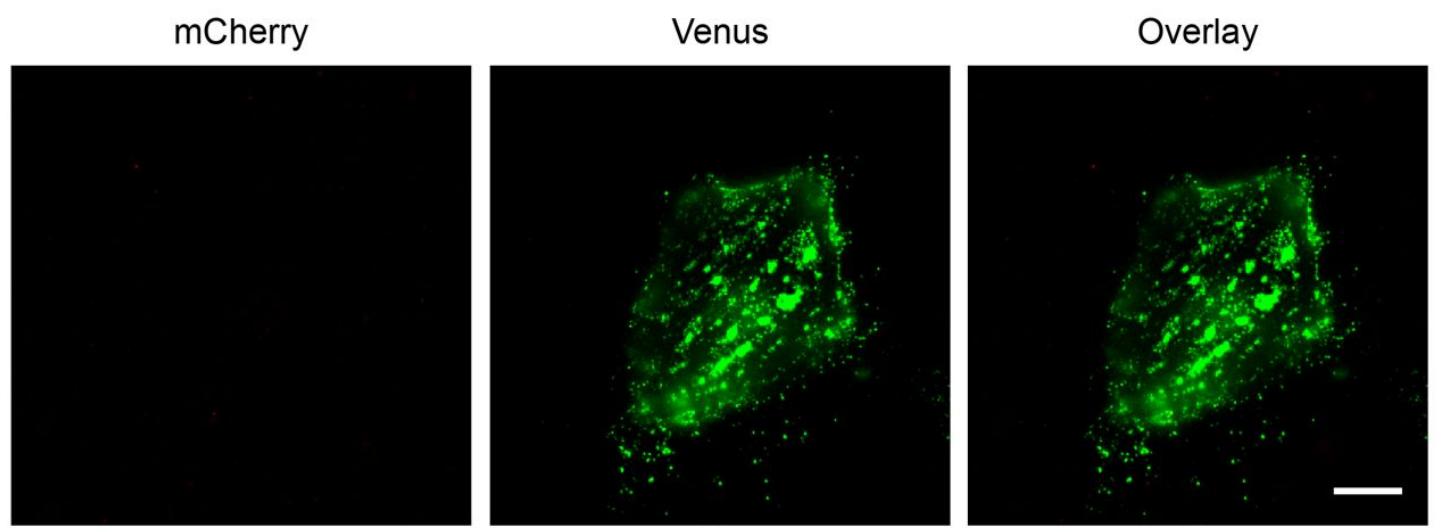

Figure S3. Widefield fluorescence imaging of HeLa cells transfected with 200ng of pCMVnl-Gag-VN plus pCMVnl-Gag-VC. Representative maximum intensity projection images of mCherry and Venus signals are shown. Scale bar, $10 \mu \mathrm{m}$. 
a pCMVnl-Gag-VN-mCherry plus pCMVnl-Gag-VC

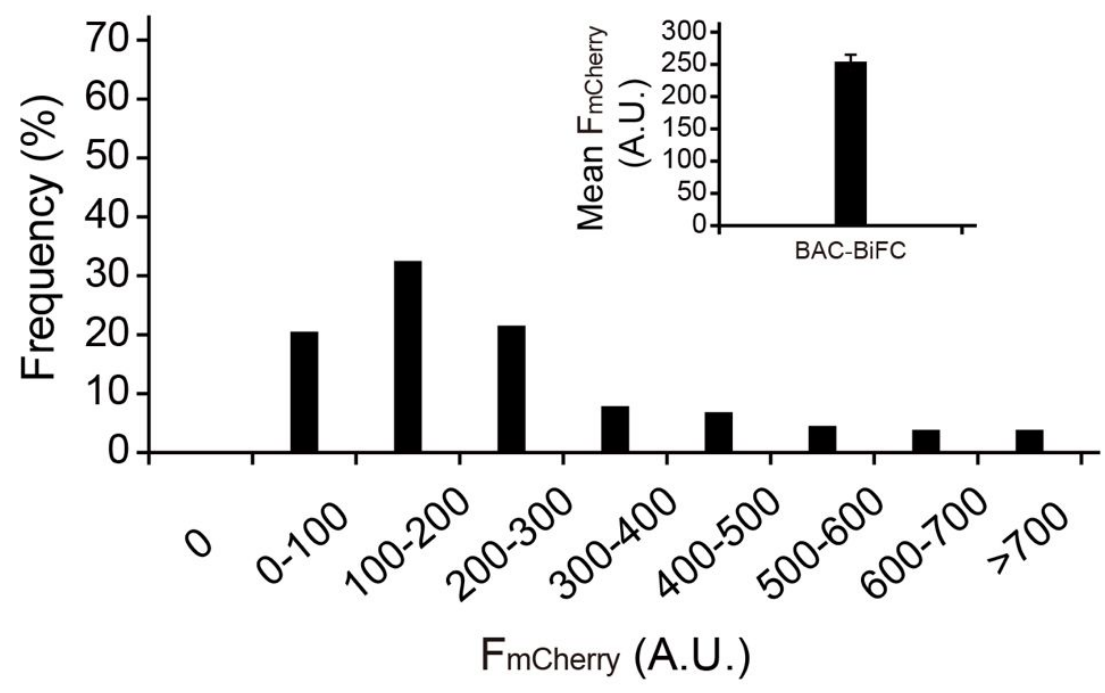

b pCMVnl-Gag-VN plus pCMVnl-Gag-VC

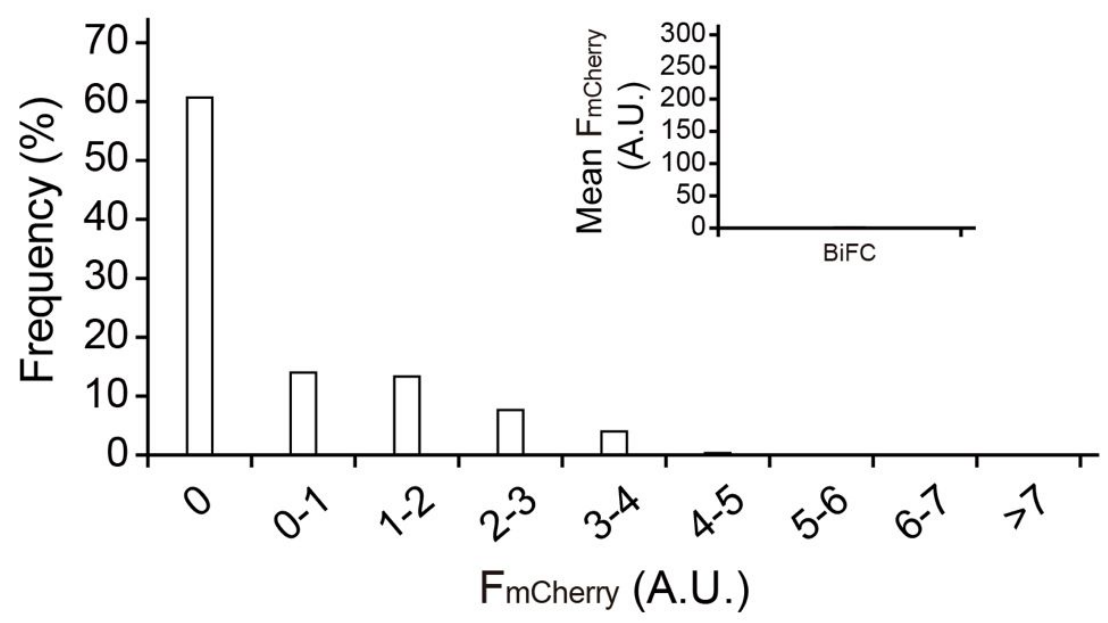

Figure $\mathbf{S 4}$. Fluorescence intensity histograms of $\mathrm{F}_{\mathrm{mCherry}}$ in HeLa cells transfected with $10 \mathrm{ng}$ of (a) pCMVnl-Gag-VN-mCherry plus pCMVnl-Gag-VC $(n=300$ cells, for BAC-BiFC imaging) or (b) pCMVnl-Gag-VN plus pCMVnl-Gag-VC ( $n=300$ cells, for BiFC imaging). Inset shows mean \pm SEM $\mathrm{F}_{\mathrm{mCherry}}$. A.U., arbitrary unit. 

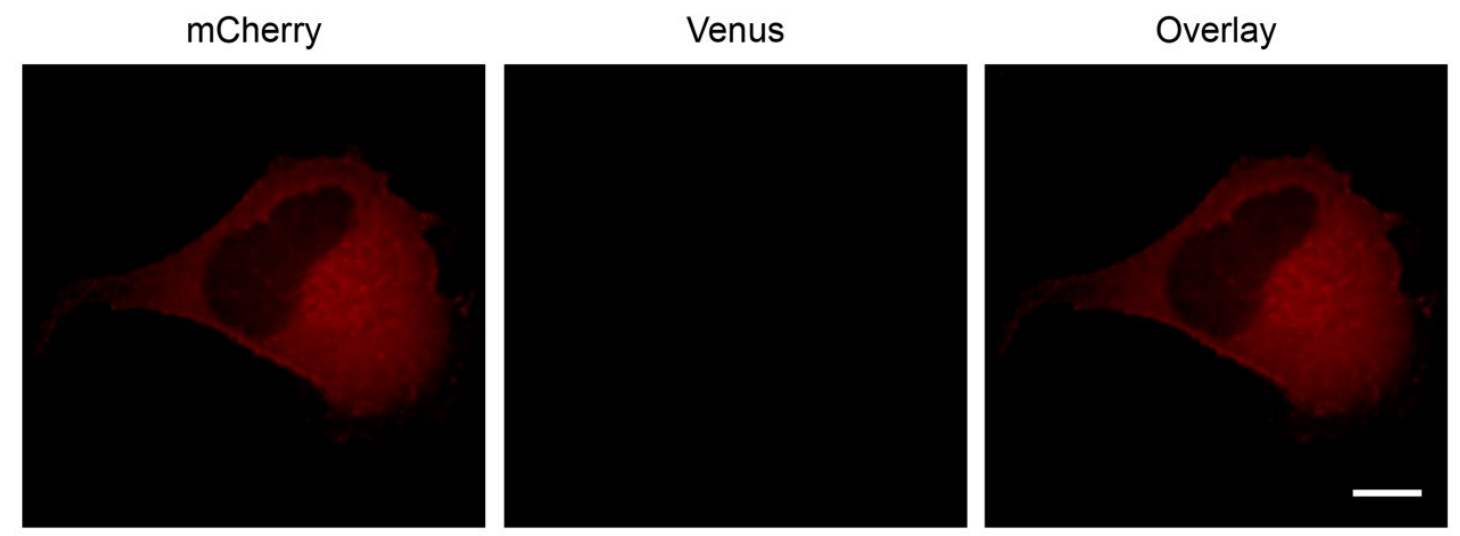

Figure S5. Widefield fluorescence imaging of HeLa cells transfected with $10 \mathrm{ng}$ of pCMVnl- $\Delta$ NC-Gag-VN-mCherry plus pCMVnl-ANC-Gag-VC. Representative maximum intensity projection images of mCherry and Venus signals are shown. Scale bar, $10 \mu \mathrm{m}$.
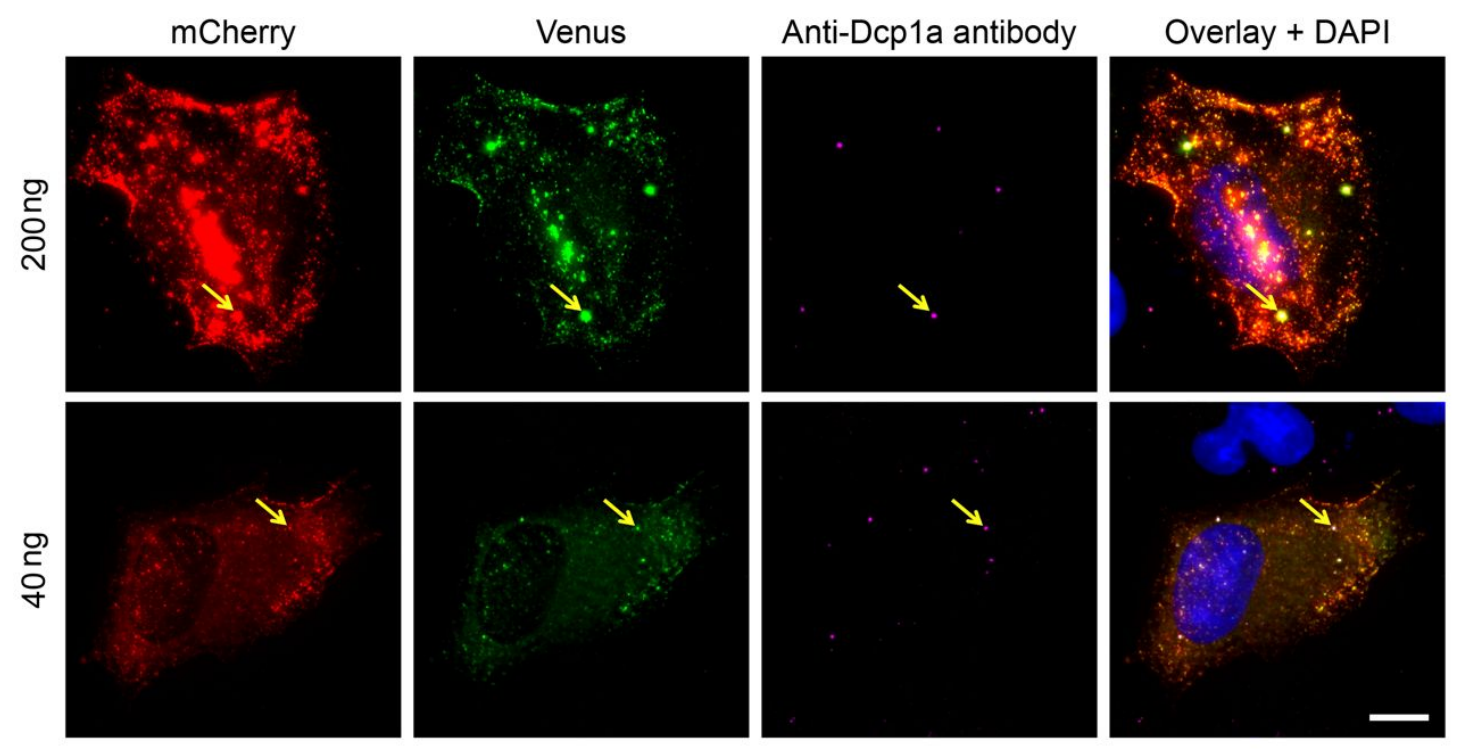

Figure S6. Representative maximum intensity projection images of mCherry, Venus and Dcp1a signals in HeLa cells transfected with $200 \mathrm{ng}$ or $40 \mathrm{ng}$ of pCMVnl-Gag-VN-mCherry plus pCMV-VC-AGO2. Dcp1a is immunostained with an anti-Dcpla antibody. The nucleus is stained with DAPI. Arrows point to the cytoplasmic foci indicative of RNA granules. Scale bar, $10 \mu \mathrm{m}$. 

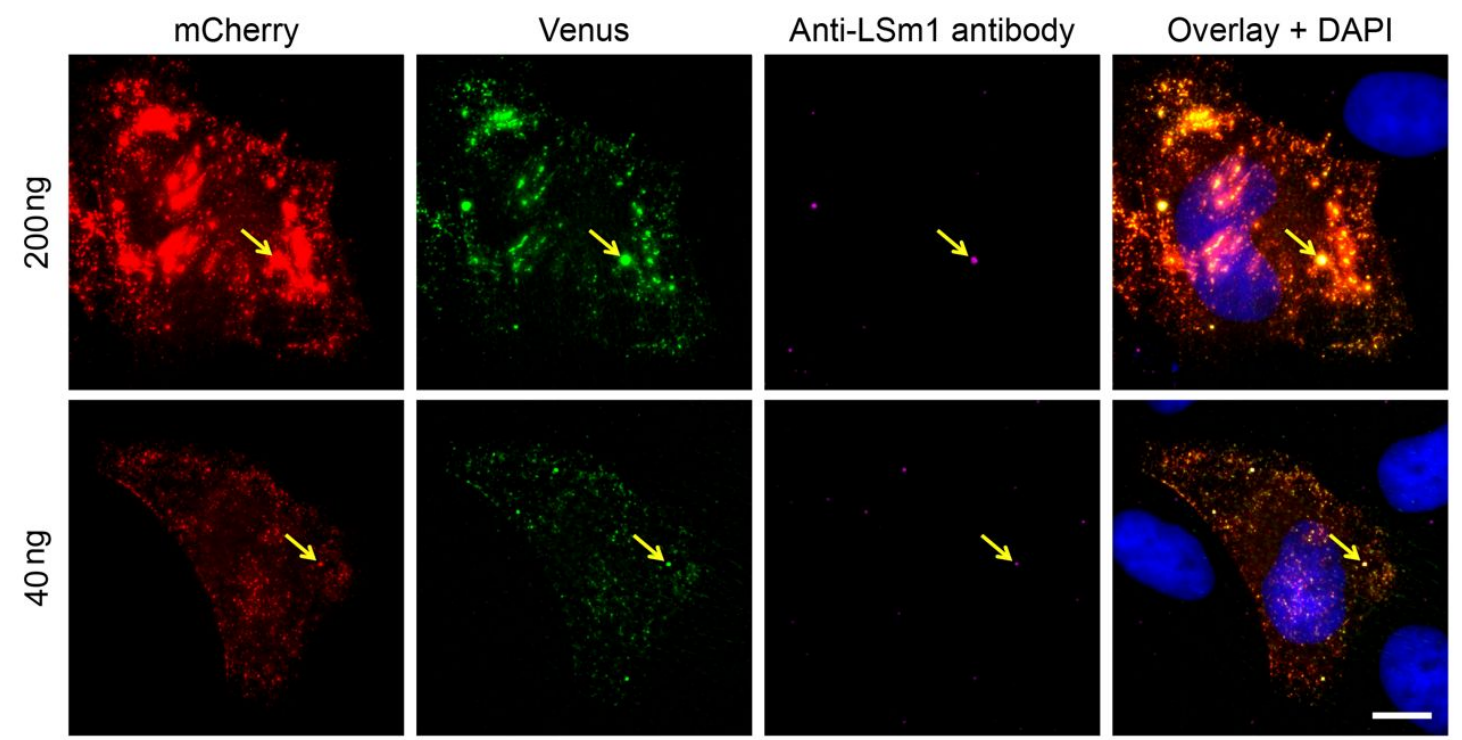

Figure S7. Representative maximum intensity projection images of mCherry, Venus and LSm1 signals in HeLa cells transfected with $200 \mathrm{ng}$ or $40 \mathrm{ng}$ of pCMVnl-Gag-VN-mCherry plus pCMV-VC-AGO2. LSm1 is immunostained with an anti-LSm1 antibody. The nucleus is stained with DAPI. Arrows point to the cytoplasmic foci indicative of RNA granules. Scale bar, $10 \mu \mathrm{m}$. 
a pCMVnl-Gag-VN-mCherry plus pCMV-VC-AGO2

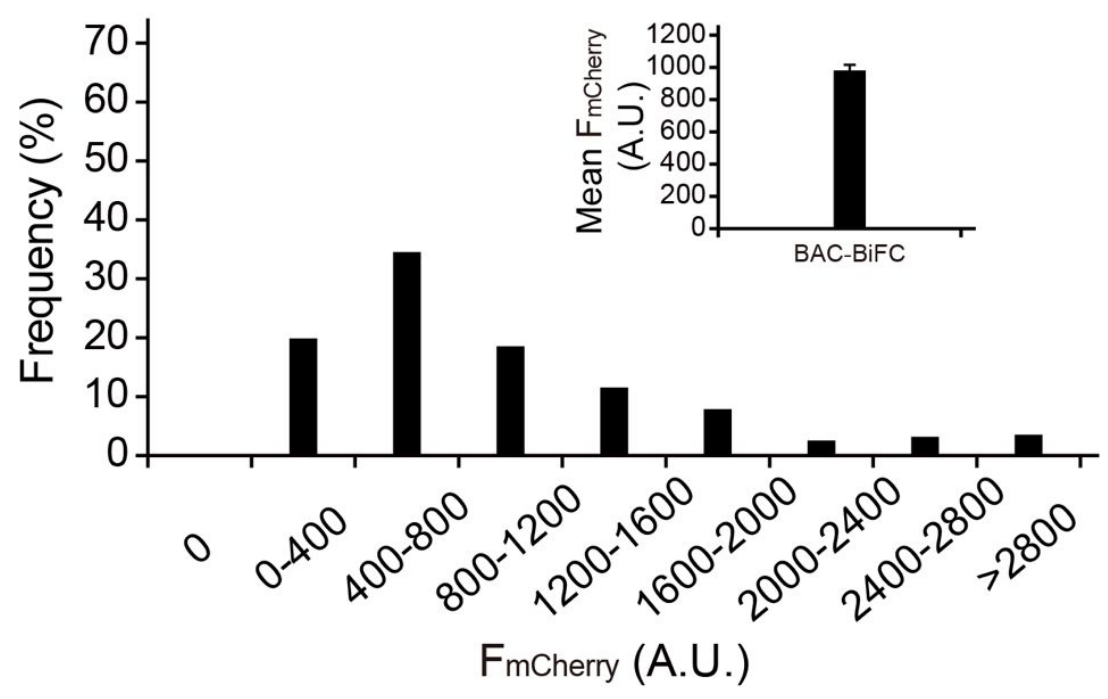

b pCMVnl-Gag-VN plus pCMV-VC-AGO2

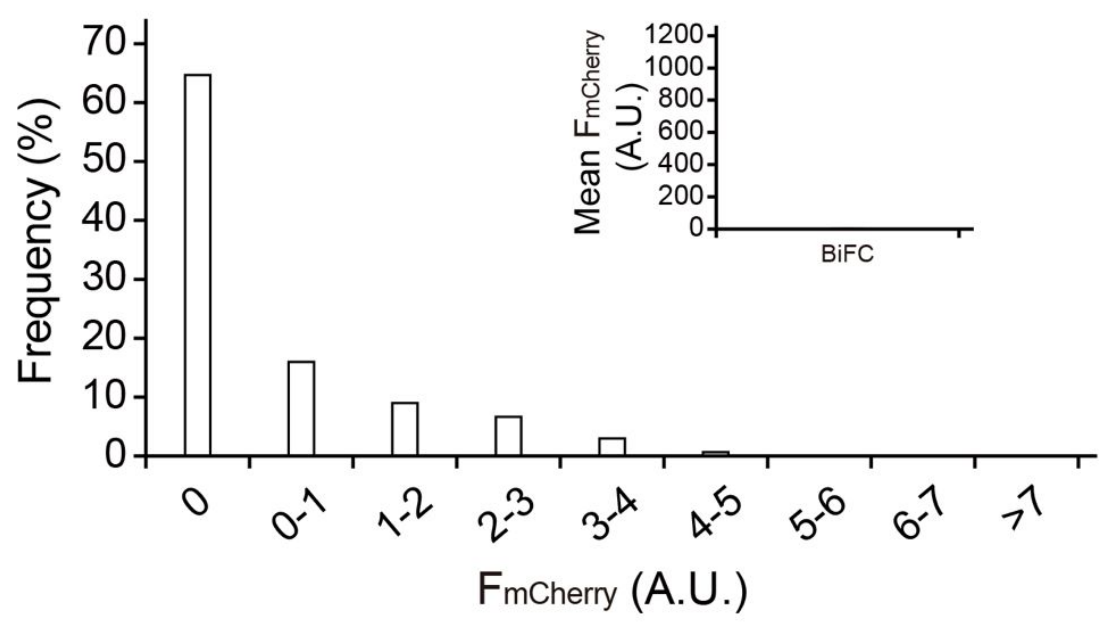

Figure S8. Fluorescence intensity histograms of $F_{m C h e r r y}$ in HeLa cells transfected with $40 \mathrm{ng}$ of (a) pCMVnl-Gag-VN-mCherry plus pCMV-VC-AGO2 $(\mathrm{n}=\mathbf{3 0 0}$ cells, for BAC-BiFC imaging) or (b) pCMVnl-Gag-VN plus pCMV-VC-AGO2 $(n=300$ cells, for BiFC imaging). Inset shows mean \pm SEM $\mathrm{F}_{\mathrm{mCherry}}$. A.U., arbitrary unit. 

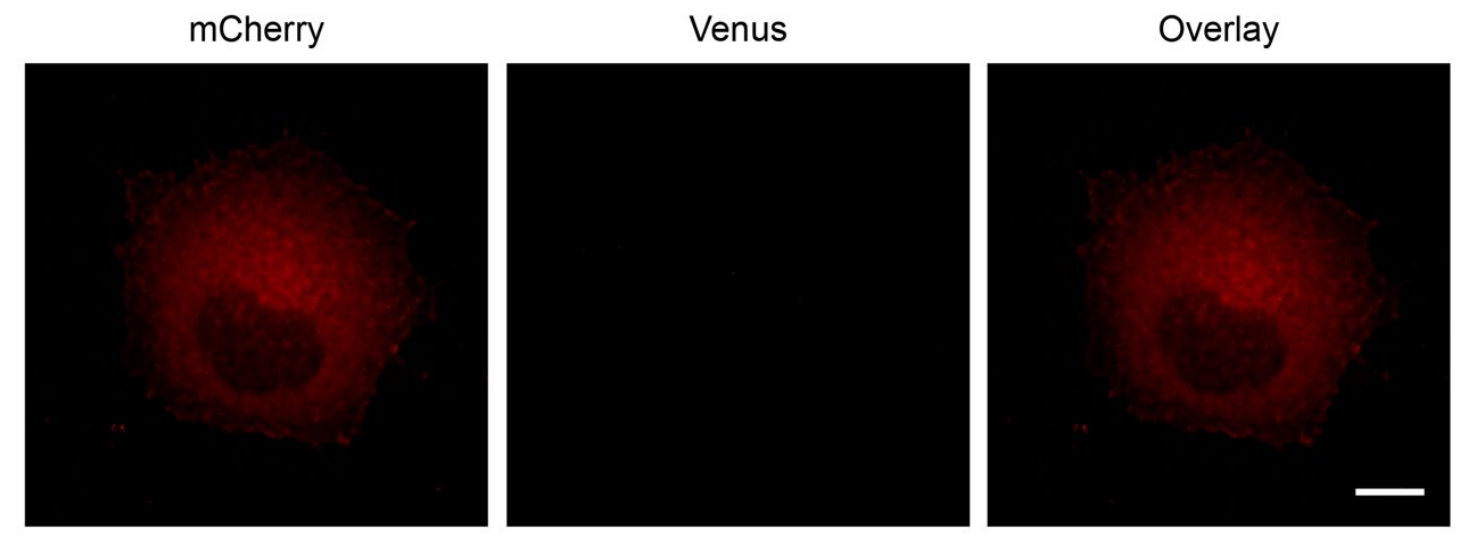

Figure S9. Widefield fluorescence imaging of HeLa cells transfected with 40ng of pCMVnl-ANC-Gag-VN-mCherry plus pCMV-VC-AGO2. Representative maximum intensity projection images of mCherry and Venus signals are shown. Scale bar, $10 \mu \mathrm{m}$.

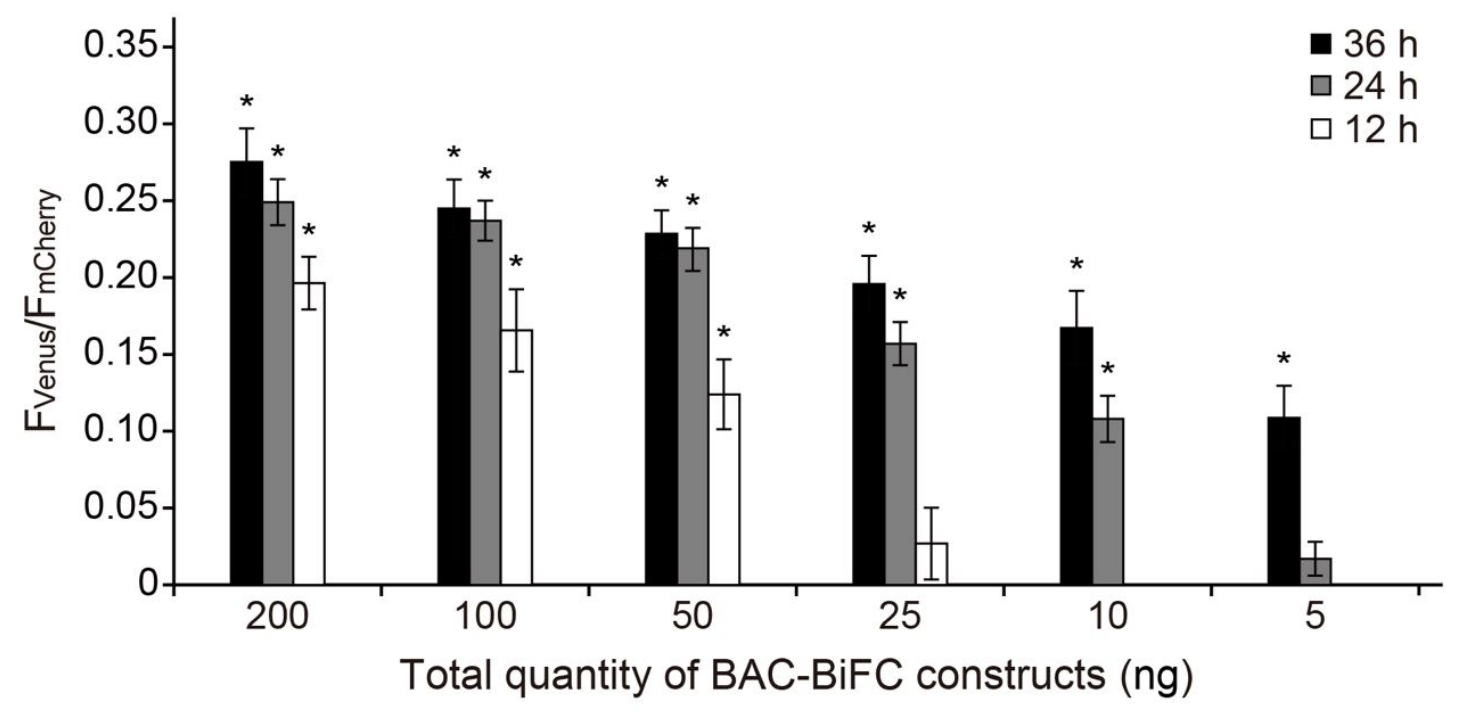

Figure S10. Ratiometric analysis of Gag-Gag interaction. HeLa cells were transfected with $200 \mathrm{ng}, 100 \mathrm{ng}, 50 \mathrm{ng}, 25 \mathrm{ng}, 10 \mathrm{ng}$ or $5 \mathrm{ng}$ of pCMVnl-Gag-VNmCherry plus pCMVnl-Gag-VC (prepared at a 1:1 weight ratio) and imaged at $12 \mathrm{~h}, 24$ $\mathrm{h}$ or $36 \mathrm{~h}$ post-transfection. $\mathrm{F}_{\text {Venus }} / \mathrm{F}_{\text {mCherry }}$ were calculated for cells under the different transfection conditions on the single-cell basis as described in MATERIALS AND METHODS. Data represent mean \pm SEM of at least 20 cells for each sample. Asterisks indicate significant differences from 0 . 